\title{
Erratum to: The Pest Species of Cicadomorpha and Fulgoromorpha (Hemiptera), Distribution and Densities in Vineyards Agroecosystems in Eastern Anatolia Region in Turkey
}

\author{
İsmail Alaserhat ${ }^{1}$ \\ Published online: 8 September 2021 \\ ๑) Springer-Verlag GmbH Deutschland, ein Teil von Springer Nature 2021
}

\section{Erratum to:}

\section{Erwerbs-Obstbau 2021}

https://doi.org/10.1007/s10341-021-00578-2

The original version of this article unfortunately contained mistakes.

Due to a technical error the botanical names are missing in the PDF of the article.

Section "Suborder Cicadomorpha" should read:

\section{Suborder Cicadomorpha}

\section{Family Aphrophoridae}

Philaenus spumarius Linnaeus, 1758

Material Examined Erzincan: 1 ( (Üzümlü, Geyikli, $39^{\circ} 42^{\prime}$ $\left.52.53^{\prime \prime} \mathrm{N}, 39^{\circ} 40^{\prime} 05.83^{\prime \prime} \mathrm{E}, 1274 \mathrm{~m}, 12 . \mathrm{IX} .2017\right)$; Erzincan: $1 \sigma^{7}$ (Üzümlü, Geyikli, 39² $42^{\prime} 50.15^{\prime \prime} \mathrm{N}, 39^{\circ} 40^{\prime} 8.01^{\prime \prime} \mathrm{E}$, 1275 m, 19.IX.2017).

\section{Family Cicadellidae}

Aconurella prolixa Lethierry, 1885

Material Examined Erzincan: 1 \& (Üzümlü, 39 $42^{\prime} 44.51^{\prime \prime} \mathrm{N}$, $\left.39^{\circ} 40^{\prime} 37.19^{\prime \prime} \mathrm{E}, 1329 \mathrm{~m}, 12 . \mathrm{IX} .2017\right)$.

The online version of the original article can be found under https://doi.org/10.1007/s10341-021-00578-2

İsmail Alaserhat

i_alaserhat36@hotmail.com

1 Directorate, Horticultural Research Institute, 24060 Erzincan, Turkey
Aphrodes makarovi Zachvatkin, 1948

Material Examined Erzincan: $10^{\top}$ (Üzümlü, 39² $42^{\prime} 42.81^{\prime \prime} \mathrm{N}$, $\left.39^{\circ} 40^{\prime} 7.23^{\prime \prime} \mathrm{E}, 1262 \mathrm{~m}, 25 . \mathrm{IX} .2014\right)$.

Arocephalus longiceps Kirschbaum, 1968

Material Examined Erzincan: 1 (Üzümlü, 39 $42^{\prime} 42.81^{\prime \prime} \mathrm{N}$, $39^{\circ} 40^{\prime} 7.23^{\prime \prime} \mathrm{E}, 1262 \mathrm{~m}$, 25.IX.2014); Erzincan: 3 $0^{\text {구 }}$ (Üzümlü, Geyikli, $39^{\circ} 42^{\prime} 50.15^{\prime \prime} \mathrm{N}, 39^{\circ} 40^{\prime} 8.01^{\prime \prime} \mathrm{E}, 1275 \mathrm{~m}$, 19.IX.2017).

Balclutha pellucens Horvath, 1909

Material Examined Erzincan: 1 (Kemah, Koçkar, 39 $34^{\prime}$ $\left.1.98^{\prime \prime} \mathrm{N}, 38^{\circ} 53^{\prime} 28.24^{\prime \prime} \mathrm{E}, 1032 \mathrm{~m}, 31 . \mathrm{VII} .2018\right)$.

Circulifer haematoceps Mulsant and Rey, 1855

Material Examined Erzincan: 2 $0^{\text {T}}+$ (Üzümlü, Geyikli, $39^{\circ} 42^{\prime} 50.15^{\prime \prime} \mathrm{N}, 39^{\circ} 40^{\prime} 8.01^{\prime \prime} \mathrm{E}, 1275 \mathrm{~m}$, 19.IX.2017).

Empoasca solani Curtis, 1846

Material Examined Erzincan: 1 ( Üzümlü, 39 $42^{\prime} 42.81^{\prime \prime} \mathrm{N}$, $39^{\circ} 40^{\prime} 7.23^{\prime \prime} \mathrm{E}, 1262 \mathrm{~m}, 26$. VIII.2014); Erzincan: $10 \mathrm{o}^{7}$ 3 q (Üzümlü, $39^{\circ} 42^{\prime} 42.81^{\prime \prime} \mathrm{N}, 39^{\circ} 40^{\prime} 7.23^{\prime \prime} \mathrm{E}, 1262 \mathrm{~m}$, 25.IX.2014); Erzincan: $10^{\top}$ (Üzümlü, Geyikli, 3942' $\left.52.53^{\prime \prime} \mathrm{N}, \quad 39^{\circ} 40^{\prime} 05.83^{\prime \prime} \mathrm{E}, 1274 \mathrm{~m}, 12 . \mathrm{IX} .2017\right)$; Erzincan: $180^{7}$ ㅇ (Üzümlü, $39^{\circ} 42^{\prime} 48.98^{\prime \prime} \mathrm{N}, 39^{\circ} 40^{\prime} 19.94^{\prime \prime} \mathrm{E}$, 1305 m, 12.IX.2017); Erzincan: 4 $\sigma^{7} 9$ (Üzümlü, Geyikli, $\left.39^{\circ} 42^{\prime} 50.15^{\prime \prime} \mathrm{N}, 39^{\circ} 40^{\prime} 8.01^{\prime \prime} \mathrm{E}, 1275 \mathrm{~m}, 19 . \mathrm{IX} .2017\right)$; Erzincan: $39 \sigma^{7}$ 古 (Üzümlü, $39^{\circ} 42^{\prime} 49.47^{\prime \prime} \mathrm{N}, 39^{\circ} 41^{\prime} 3.68^{\prime \prime} \mathrm{E}$, 1399 m, 19.IX.2017); Erzincan: 2 ơ 9 (Kemah, Boğaziçi, $39^{\circ} 34^{\prime} 1.91^{\prime \prime} \mathrm{N}, 38^{\circ} 53^{\prime} 36.14^{\prime \prime} \mathrm{E}, 1030 \mathrm{~m}, 31 . \mathrm{VII} .2018$ ); Tunceli: $20^{\top}$ ○ (Çemişgezek, $39^{\circ} 4^{\prime} 24.03^{\prime \prime} \mathrm{N}, 38^{\circ} 54^{\prime} 57.44^{\prime \prime} \mathrm{E}$, 
1074 m, 27.VIII.2019); Tunceli: 2 o $^{7}$ (Mazgirt, Akpazar, $38^{\circ} 51^{\prime} 42.90^{\prime \prime} \mathrm{N}, 39^{\circ} 46^{\prime} 47.54^{\prime \prime} \mathrm{E}, 855 \mathrm{~m}, 28$.VIII.2019).

Euscelis incisus Kirschbaum, 1868

Material Examined Erzincan: 2 (Üzümlü, $39^{\circ} 42^{\prime} 42.81^{\prime \prime} \mathrm{N}$, $39^{\circ} 40^{\prime} 7.23^{\prime \prime} \mathrm{E}, 1262 \mathrm{~m}$, 05.VIII.2014); Erzincan: $20^{7}$ 4 ㅇ (Üzümlü, $39^{\circ} 42^{\prime} 42.81^{\prime \prime} \mathrm{N}, 39^{\circ} 40^{\prime} 7.23^{\prime \prime} \mathrm{E}, 1262 \mathrm{~m}$, 26.VIII.2014); Erzincan: 1 † (Üzümlü, $39^{\circ} 42^{\prime} 42.81^{\prime \prime} \mathrm{N}$, $39^{\circ} 40^{\prime} 7.23^{\prime \prime}$ E, 1262 m, 25.IX.2014); Erzincan: 2 甲 (Kemah, Boğaziçi, $\quad 39^{\circ} 34^{\prime} 1.91^{\prime \prime} \mathrm{N}, \quad 38^{\circ} 53^{\prime} 36.14^{\prime \prime} \mathrm{E}, \quad 1030 \mathrm{~m}$, 31.VII.2018).

\section{Euscelis lineolatus Brullé, 1832}

Material Examined Tunceli: $3 \sigma^{7} q$ (Burmageçit, 38 $57^{\prime}$ 56.52" N, 39 31'36.31" E, 996m, 28.VIII.2019); Tunceli: 1 ㅇ (Çemişgezek, 39 $4^{\prime} 24.03^{\prime \prime} \mathrm{N}, 38^{\circ} 54^{\prime} 57.44^{\prime \prime} \mathrm{E}, 1074 \mathrm{~m}$, 27.VIII.2019); Tunceli: $30^{7}$ ㅇ (Mazgirt, Akpazar, 38 51' $42.90^{\prime \prime} \mathrm{N}, 39^{\circ} 46^{\prime} 47.54^{\prime \prime} \mathrm{E}, 855 \mathrm{~m}$, 28.VIII.2019).

Hauptidia cretacea Dlabola, 1957

Material Examined Erzincan: 1 ( (Üzümlü, 39² $42^{\prime} 48.98^{\prime \prime} \mathrm{N}$, 39 $\left.40^{\prime} 19.94^{\prime \prime} \mathrm{E}, 1305 \mathrm{~m}, 12 . \mathrm{IX} .2017\right)$; Erzincan: 2 ㅇ (Kemaliye, Çit, $39^{\circ} 7^{\prime} 3.31^{\prime \prime} \mathrm{N}, \quad 38^{\circ} 35^{\prime} 3.51^{\prime \prime} \mathrm{E}, \quad 1024 \mathrm{~m}$, 14.VIII.2018); Tunceli: $320^{\circ}$ ' (Burmageçit, $38^{\circ} 57^{\prime} 56.52^{\prime \prime} \mathrm{N}$, 39 $31^{\prime} 36.31^{\prime \prime} \mathrm{E}, 996 \mathrm{~m}, 28$.VIII.2019); Tunceli: 3 o $^{7}$ ㅇ (Mazgirt, Akpazar, $38^{\circ} 51^{\prime} 42.90^{\prime \prime} \mathrm{N}, 39^{\circ} 46^{\prime} 47.54^{\prime \prime} \mathrm{E}, 855 \mathrm{~m}$, 28.VIII.2019).

Macrosteles quadripunctulatus Kirschbaum, 1868

Material Examined Erzincan: $20^{\top} \bigcirc$ (Üzümlü, 39 $42^{\prime}$

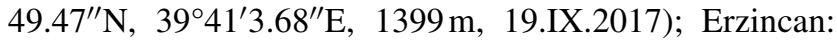

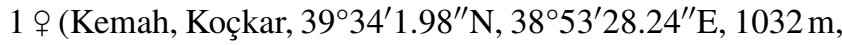
31.VII.2018).

Micantulina micantula Zetterstedt, 1840

Material Examined Erzincan: 1 ( Üzümlü, $39^{\circ} 42^{\prime} 42.81^{\prime \prime} \mathrm{N}$, $39^{\circ} 40^{\prime} 7.23^{\prime \prime} \mathrm{E}, 1262 \mathrm{~m}, 05$. VIII.2014).

Phlepsius intricatus Herrich-Schaffer, 1838

Material Examined Erzincan: $10^{\top}$ (Üzümlü, $39^{\circ} 42^{\prime} 42.81^{\prime \prime} \mathrm{N}$, $39^{\circ} 40^{\prime} 7.23^{\prime \prime} \mathrm{E}, 1262 \mathrm{~m}, 26$. VIII.2014).

Platymetopius manfredi Abdul-Nour, 1987

Material Examined Erzincan: $20^{\top}$ (Üzümlü, $39^{\circ} 42^{\prime} 42.81^{\prime \prime} \mathrm{N}$, $39^{\circ} 40^{\prime} 7.23^{\prime \prime} \mathrm{E}, 1262 \mathrm{~m}, 26$. VIII.2014).
Psammotettix alienus Dahlbom, 1850

Material Examined Erzincan: 5 ơ $0^{\text {T }}$ (Üzümlü, Geyikli, $39^{\circ} 42^{\prime} 50.15^{\prime \prime} \mathrm{N}, 39^{\circ} 40^{\prime} 8.01^{\prime \prime} \mathrm{E}, 1275 \mathrm{~m}$, 19.IX.2017); Erz-

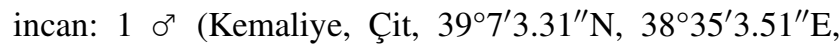
$1024 \mathrm{~m}, 14$.VIII.2018).

Psammotettix confinis Dahlbom, 1850

Material Examined Erzincan: $60^{7} \bigcirc$ (Üzümlü, 39² $42^{\prime}$ $49.47^{\prime \prime} \mathrm{N}, 39^{\circ} 41^{\prime} 3.68^{\prime \prime} \mathrm{E}, 1399 \mathrm{~m}$, 19.IX.2017).

Psammotettix provincialis Ribaut, 1925

Material Examined Tunceli: $40^{7}$ (Çemişgezek, 39 $4^{\circ}$ $\left.24.03^{\prime \prime} \mathrm{N}, 38^{\circ} 54^{\prime} 57.44^{\prime \prime} \mathrm{E}, 1074 \mathrm{~m}, 27 . \mathrm{VIII} .2019\right)$; Tunceli: $1 \sigma^{7}$ (Burmageçit, 38 $57^{\prime} 56.52^{\prime \prime} \mathrm{N}, 39^{\circ} 31^{\prime} 36.31^{\prime \prime} \mathrm{E}, 996 \mathrm{~m}$, 28.VIII.2019); Tunceli: $10^{7}$ (Mazgirt, Akpazar, 38 $51^{\prime}$ $\left.42.90^{\prime \prime} \mathrm{N}, 39^{\circ} 46^{\prime} 47.54^{\prime \prime} \mathrm{E}, 855 \mathrm{~m}, 28 . \mathrm{VIII} .2019\right)$; Erzincan: $110^{7}+$ (Kemah, Koçkar, 39 $34^{\prime} 1.98^{\prime \prime} \mathrm{N}, 38^{\circ} 53^{\prime} 28.24^{\prime \prime} \mathrm{E}$, 1032 m, 31.VII.2018).

Rhopalopyx vitripennis Flor, 1861

Material Examined Erzincan: $30^{7}$ ㅇ (Üzümlü, 394 $42^{\prime}$ $49.47^{\prime \prime} \mathrm{N}, 39^{\circ} 41^{\prime} 3.68^{\prime \prime} \mathrm{E}, 1399 \mathrm{~m}$, 19.IX.2017).

Stenometopiellus angorensis Zachvatkin, 1946

Material Examined Erzincan: $40^{7} \bigcirc$ (Kemah, Koçkar, $39^{\circ} 34^{\prime} 1.98^{\prime \prime} \mathrm{N}, 38^{\circ} 53^{\prime} 28.24^{\prime \prime} \mathrm{E}, 1032 \mathrm{~m}, 31$.VII.2018).

Zyginidia sohrab Zachvatkin, 1947

Material Examined Erzincan: 1 (Üzümlü, $39^{\circ} 42^{\prime} 48.98^{\prime \prime} \mathrm{N}$, $\left.39^{\circ} 40^{\prime} 19.94^{\prime \prime} \mathrm{E}, 1305 \mathrm{~m}, 12 . \mathrm{IX} .2017\right)$; Erzincan: $1 \mathrm{o}^{\prime \prime}$ (Üzümlü, $\quad 39^{\circ} 42^{\prime} 44.51^{\prime \prime} \mathrm{N}, \quad 39^{\circ} 40^{\prime} 37.19^{\prime \prime} \mathrm{E}, \quad 1329 \mathrm{~m}$, 12.IX.2017); Erzincan: 4 ơ $q$ (Üzümlü, $39^{\circ} 42^{\prime} 49.47^{\prime \prime} \mathrm{N}$, $\left.39^{\circ} 41^{\prime} 3.68^{\prime \prime} \mathrm{E}, 1399 \mathrm{~m}, 19 . \mathrm{IX} .2017\right)$.

\section{Family Membracidae}

Stictocephala bisonia Kopp and Yonke, 1977

Material Examined Erzincan: 2 ơ + (Üzümlü, Geyikli, $\left.39^{\circ} 42^{\prime} 52.53^{\prime \prime} \mathrm{N}, 39^{\circ} 40^{\prime} 05.83^{\prime \prime} \mathrm{E}, 1274 \mathrm{~m}, 12 . I X .2017\right)$; Erzincan: 1 q (Üzümlü, $39^{\circ} 42^{\prime} 49.47^{\prime \prime} \mathrm{N}, 39^{\circ} 41^{\prime} 3.68^{\prime \prime} \mathrm{E}, 1399 \mathrm{~m}$, 19.IX.2017). 


\section{Suborder Fulgoromorpha}

\section{Family Delphacidae}

Laodelphax striatellus Fallen, 1826

Material Examined Erzincan: 1 ( Üzümlü, $39^{\circ} 42^{\prime} 42.81^{\prime \prime} \mathrm{N}$, $39^{\circ} 40^{\prime} 7.23^{\prime \prime} \mathrm{E}, 1262 \mathrm{~m}$, 05.VIII.2014); Erzincan: 1 ㅇ, $\mathrm{o}^{\top}$ (Üzümlü, $39^{\circ} 42^{\prime} 42.81^{\prime \prime} \mathrm{N}, \quad 39^{\circ} 40^{\prime} 7.23^{\prime \prime} \mathrm{E}, \quad 1262 \mathrm{~m}$, 26.VIII.2014); Erzincan: 1 ㅇ, $0^{\prime \prime}$ (Üzümlü, $39^{\circ} 42^{\prime} 42.81^{\prime \prime} \mathrm{N}$, $\left.39^{\circ} 40^{\prime} 7.23^{\prime \prime} \mathrm{E}, 1262 \mathrm{~m}, 25 . \mathrm{IX} .2014\right)$; Erzincan: 3 ․, (Kemah, Koçkar, 39 $34^{\prime} 1.98^{\prime \prime}$ N, 38 $8^{\circ} 3^{\prime} 28.24^{\prime \prime} \mathrm{E}, 1032 \mathrm{~m}$, 31.VII.2018).

Toya propinqua Fieber, 1866

Material Examined Erzincan: 1 (Üzümlü, $39^{\circ} 42^{\prime} 48.97^{\prime \prime} \mathrm{N}$, $\left.39^{\circ} 40^{\prime} 19.97^{\prime \prime} \mathrm{E}, 1302 \mathrm{~m}, 12 . \mathrm{IX} .2017\right)$.

\section{Family Dictyopharidae}

Dictyophara europaea Linnaeus, 1767

Material Examined Erzincan: 1 व (Üzümlü, Geyikli, $\left.39^{\circ} 42^{\prime} 52.53^{\prime \prime} \mathrm{N}, 39^{\circ} 40^{\prime} 05.83^{\prime \prime} \mathrm{E}, 1274 \mathrm{~m}, 12 . I X .2017\right)$.

Dictyophara multireticulata Mulsant and Rey, 1855

Material Examined Erzincan: $1 \sigma^{\prime \prime}$ (Üzümlü, 394 $42^{\prime} 42.81^{\prime \prime} \mathrm{N}$, $\left.39^{\circ} 40^{\prime} 7.23^{\prime \prime} \mathrm{E}, 1262 \mathrm{~m}, 05 . \mathrm{VIII} .2014\right)$.

In this article the two following references were missing:

Anonymous (2017) Bağ Entegre Mücadele Teknik Talimatları. Ministry of Agriculture and Forestry, General Directorate of Agricultural Research and Policies Publications, 120 p, Ankara-Turkey (in Turkish).

Anonymous (2018) FAO (Food and Agriculture Organization of the United Nations). http://www.fao.org/faostat/ en/\#data/QC Accessed 01 Dec 2020.

The original article has been corrected. 